\title{
The Effect of Prosthesis Design on Blood Loss in Simultaneous Bilateral Total Knee Arthroplasty: Closed-Box versus Open-Box Prosthesis
}

\author{
Piti Rattanaprichavej, MD, Artit Laoruengthana, MD, Supachok Rasamimogkol, MD, \\ Panapol Varakornpipat, MD, Kongpob Reosanguanwong, MD, Krit Pongpirul, MD* \\ Department of Orthopedics, Faculty of Medicine, Naresuan University, Phitsanulok, \\ *Department of Preventive and Social Medicine, Faculty of Medicine, Chulalongkorn University, Bangkok, Thailand
}

\begin{abstract}
Background: Various pre- and perioperative risk factors have been reported in association with blood loss in knee arthroplasty. However, the effect of the uncovered cancellous surface on blood loss in simultaneous bilateral total knee arthroplasty (SBTKA) by different prosthetic designs is not well elucidated. Therefore, this study aimed to compare the blood loss and transfusion rate between different knee prostheses in SBTKA and to identify risk factors that influence blood loss and transfusion after SBTKA.

Methods: Demographic and perioperative data of patients who underwent SBTKA using either a closed-box or an open-box femoral component of posterior-stabilized fixed-bearing (PS FB) knee system were retrospectively reviewed. The calculated blood loss (CBL) and blood transfusion rate were compared by using Student $t$-test and confirmed with multivariate regression analysis.

Results: There was no significant difference in preoperative parameters between 54 closed-box and 56 open-box PS FB TKAs. The CBL of the closed-box TKA group was $135.23 \mathrm{~mL}$ less (95\% confidence interval [CI], -215.30 to $-55.16 ; p=0.001$ ) than that of the open-box TKA group. However, the blood transfusion rates of the closed- and open-box TKA groups were not significantly different (24.1\% and $38.5 \%, p=0.11)$. For each additional minute of total operative time, $3.75 \mathrm{~mL}(95 \% \mathrm{Cl}, 1.75$ to $5.76 ; p<0.001$ ) of blood loss was anticipated. For each additional $\mathrm{mg} / \mathrm{dL}$ of preoperative hemoglobin, $71 \%(p<0.001)$ reduction of blood transfusion probability was predicted.

Conclusions: The use of closed- and open-box knee prostheses resulted in a significant difference in blood loss in SBTKA. Prolonged operative time also significantly increased CBL. Therefore, strategies to control the bleeding surface and shorten operative duration may be considered if blood loss is of special concern. The preoperative hemoglobin was the only factor that affects the probability of blood transfusion in SBTKA.
\end{abstract}

Keywords: Knee, Arthroplasty, Prosthesis, Bilateral, Blood loss, Transfusion

Total knee arthroplasty (TKA) is a successful surgical procedure for restoring knee joint function and relieving pain in end-stage knee arthropathy. Approximately $20 \%$ of pa-

Received December 23, 2018; Accepted March 25, 2019

Correspondence to: Piti Rattanaprichavej, MD

Department of Orthopedics, Faculty of Medicine, Naresuan University, 99 Moo 9 Thapho, Phitsanulok 65000, Thailand

Tel: +66-869966995, Fax: +66-55965105

E-mail: pt-rp@hotmail.com tients have severe pain on both knees and $10 \%$ of patients undergo contralateral TKA within 1 year after the first procedure. ${ }^{1)}$ Therefore, simultaneous bilateral TKA (SBT$\mathrm{KA}$ ) could provide advantages including the single operation, shortened total recovery time, and reduced perioperative cost. However, significantly higher perioperative morbidity has been demonstrated after SBTKA than after unilateral TKA (UTKA). ${ }^{2,3)}$ One of the major concerns in SBTKA is that the procedure can lead to a more substantial blood loss because of large exposure, deep soft-tissue dissection, and sinus bleeding from cancellous and uncov- 
Rattanaprichavej et al. Closed- versus Open-Box Prosthesis

Clinics in Orthopedic Surgery • Vol. 11, No. 4, $2019 \bullet$ www.ecios.org

ered surfaces after bone resection, ${ }^{4-7)}$ resulting in a transfusion rate of $44.7 \%$ to $55 \%{ }^{8.9)}$ Although blood transfusion is the standard management of blood loss, it can increase the risk of complications such as cardiovascular volume overload, blood-transmitted disease, allergic reaction, and periprosthetic joint infection. ${ }^{10,11)}$ Various pre- and perioperative risk factors including age, sex, race, body mass index (BMI), preoperative hemoglobin ( $\mathrm{Hb}$ ) level, comorbidity, and duration of operation have been reported in association with blood loss and postoperative blood transfusion in UTKA, ${ }^{8,12,13)}$ and these factors might be assumed to be implicated in the bilateral procedure. However, there are few studies that investigated the significance of these potential determinants in SBTKA.

Additionally, evidence is sparse on the effect of different knee prosthetic designs on perioperative blood loss. The posterior-stabilized (PS) and cruciate-retaining (CR) designs of a similar knee prosthesis model were reported to result in different blood loss following UTKA. ${ }^{7,14)}$ The housing area of the distal femoral bone cut in PS TKA was supposed to be the source of bleeding and increasing perioperative blood loss. However, there was no difference in blood transfusion between the knee prosthetic designs. ${ }^{7,14)}$ As stated above, SBTKA will cause more substantial blood loss; thus, the different knee prostheses may have more influence on blood loss and the requirement for blood transfusion in the bilateral procedure. At our institution, we have implanted two different designs of PS fixed-bearing (PS FB) femoral prostheses in TKA: closed-box design and open-box design that covers and uncovers cancellous bone of the distal femoral housing, respectively. However, the benefit of the closed-box femoral component in SBTKA is still unclear. Hence, this study aimed to compare the drain output (DO), calculated blood loss (CBL), and allogeneic
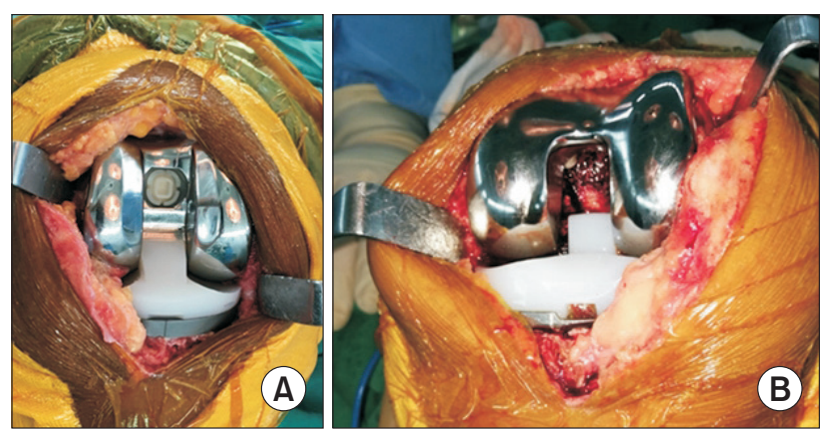

Fig. 1. (A) The closed-box prosthesis fully covers cancellous bone of the distal femoral housing. (B) Bleeding from the area of trimmed cancellous surface may be responsible for the significantly higher calculated blood loss in the open-box prosthesis group than that in the closed-box prosthesis group. blood transfusion rate between SBTKAs that implanted different knee designs; and to identify the risk factors that may be associated with CBL and blood transfusion. We hypothesized that implantation of the less exposed trimmed-cancellous surface (closed box) may reduce blood loss when compared to the more exposed cancellous bone (open box), particularly in SBTKA (Fig. 1).

\section{METHODS}

We retrospectively analyzed the prospectively collected data of 152 patients who underwent SBTKA using either the closed-box or open-box femoral prosthesis design from June 2015 to May 2018. Four patients with preoperative $\mathrm{Hb}$ less than $10 \mathrm{~g} / \mathrm{dL}, 13$ patients with incomplete data, and 25 patients operated with accelerometer-based navigation were excluded. A total of 110 SBTKA patients (54 closed-box and 56 open-box TKAs) remained for assessment (Fig. 2). The study was approved by the Institutional Review Board of Naresuan University with the study registry of TCTR20180220001.

All surgical procedures were performed by a single surgeon $(\mathrm{AL})$ with an identical surgical technique through a standard medial parapatellar approach. All the patients underwent regional anesthesia and received a periarticular injection with a multidrug mixture $(0.5 \%$ Marcaine, $20 \mathrm{~mL}$ [AstraZeneca, Lund, Sweden], 30 mg Ketorolac [Siu Guan, Chiayi, Taiwan], $5 \mathrm{mg}$ morphine sulfate, and $300 \mu \mathrm{g}$ adrenaline $[1: 1000,0.3 \mathrm{~mL}])$. The procedure was done under tourniquet control of $250 \mathrm{mmHg}$ by inflating before the incision. Following an arthrotomy, a distal femoral cut was performed with an intramedullary guide system while a proximal tibial cut was done with an extramedullary guide system. After completion of the bone cut, the opening of the femoral medullary canal was closed with a bone plug.

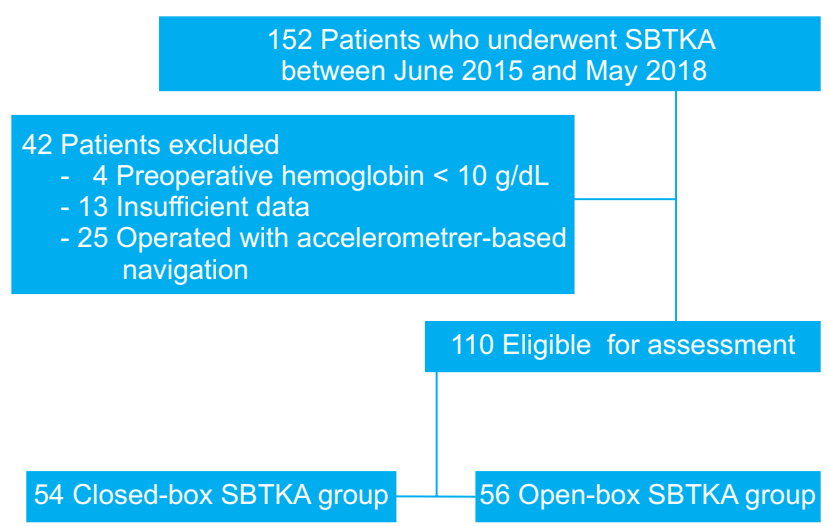

Fig. 2. Study flowchart. SBTKA: simultaneous bilateral total knee arthroplasty. 
All the knees were implanted with a cemented PS FB TKA system. There were two types of knee prosthesis used during the study period: closed-box TKA (PFC Sigma; DePuy Synthes, Warsaw, IN, USA) and open-box TKA (NexGen LPS; Zimmer Biomet, Warsaw, IN, USA). The patella was resurfaced in all cases of the closed-box group but in none of the cases in the open-box group. A vacuum drain was applied, and then $15 \mathrm{mg} / \mathrm{kg}$ of topical tranexamic acid (TXA) was poured into the knee joint before closure of the arthrotomy. A compressive dressing was applied and the tourniquet was deflated. The drains were closed for 3 hours in all knees.

An identical postoperative protocol was conducted for every patient. During the first 48 hours, intravenous (IV) patient-controlled analgesia morphine (on-demand bolus of $0.5 \mathrm{mg}$ of morphine sulfate with a 5 -minute lockout period) and $30 \mathrm{mg}$ of ketorolac every 8 hours were administered for pain control. After 48 hours, all the catheters and drains were discarded, and then $250 \mathrm{mg}$ of naproxen was given every 12 hours orally combined with additional $2 \mathrm{mg}$ of IV morphine as rescue analgesia for breakthrough pain. The serum $\mathrm{Hb}$ level was monitored at 24,48 , and 72 hours after the index surgery. The patients began postoperative physiotherapy on the day after surgery. Postoperative thromboembolic prophylaxis consisted of low-molecular-weight heparin injected subcutaneously daily for 2 days and oral warfarin for 10 days.

The outcome parameters including demographic data, total operative time (TOT), DO, postoperative $\mathrm{Hb}$, blood transfusion rate, and length of stay (LOS) were routinely recorded by the independent physicians (PV, KR) at our institution. The total blood volume (TBV) and CBL were calculated by using the equation of Nadler et al. ${ }^{15}$ ) and the $\mathrm{Hb}$ balance method, ${ }^{16)}$ respectively:

Male TBV $(\mathrm{mL})=\left(0.0003669 \times\right.$ height $\left.^{3}[\mathrm{~cm}]\right)+$
$(32.19 \times$ body weight $[\mathrm{kg}])+604$

Female TBV $(\mathrm{mL})=\left(0.0003561 \times\right.$ height $\left.^{3}[\mathrm{~cm}]\right)+$ $(33.08 \times$ body weight $[\mathrm{kg}])+183$, and

$\mathrm{CBL}(\mathrm{mL})=\mathrm{TBV}(\mathrm{mL}) \times\left(\mathrm{Hb}_{\mathrm{i}}-\mathrm{Hb}_{\mathrm{e}}\right) / \mathrm{Hb}_{\mathrm{i}}+$ sum of blood products transfused $(\mathrm{mL})$,

where $\mathrm{Hb}_{\mathrm{i}}(\mathrm{g} / \mathrm{dL})$ was defined as the preoperative $\mathrm{Hb} ; \mathrm{Hb}_{\mathrm{e}}(\mathrm{g} / \mathrm{dL})$, as the postoperative $\mathrm{Hb}$.

The difference between the preoperative and lowest $\mathrm{Hb}$ was applied to the $\mathrm{Hb}$ balance method. The blood transfusion threshold for our SBTKA procedure is postoperative $\mathrm{Hb}$ level below $9.0 \mathrm{~g} / \mathrm{dL}$.

\section{Statistical Analysis}

Descriptive statistics were used as appropriate. The unpaired Student $t$-test was used to compare the difference of measured outcomes (DO, CBL, TOT, and LOS) between the two groups. Blood transfusion rate was compared between the two groups by using chi-square test. Multivariate regression analysis, controlling for age, BMI, American Society of Anesthesiologist (ASA) physical status classification, preoperative $\mathrm{Hb}$, TOT, and type of knee prosthesis, was used for analysis of factors associated with the CBL and blood transfusion rate. Statistical significance was defined as $p<0.05$. Stata/MP ver. 15.0 (Stata Corp., College Station, TX, USA) was used for all analyses. A post hoc analysis revealed that the sample size of this study had $88.2 \%$ power to detect a difference in the mean CBL of 120 $\mathrm{mL}$ with a standard deviation of $250 \mathrm{~mL}$ and an alpha of 0.05 .

\section{RESULTS}

There was no significant difference between two groups (54 closed-box and 56 open-box TKAs) in terms of demographics and preoperative $\mathrm{Hb}$ level (Table 1). The TOT

Table 1. Demographic Characteristics

\begin{tabular}{lccc}
\multicolumn{1}{c}{ Variable } & Closed-box SBTKA & Open-box SBTKA & $p$-value* \\
\hline Female:male & $53: 1$ & $53: 3$ & - \\
\hline Age $(\mathrm{yr})$ & $61.7 \pm 7.2(51-86)$ & $62.2 \pm 7.0(45-75)$ & 0.696 \\
\hline BMI $\left(\mathrm{kg} / \mathrm{m}^{2}\right)$ & $26.1 \pm 5.7(18.8-37.9)$ & $26.8 \pm 3.6(18.4-43.3)$ & 0.489 \\
\hline ASA $(1: 2: 3)$ & $3: 43: 8$ & $4: 38: 14$ & 0.331 \\
\hline Preoperative hemoglobin $(\mathrm{mg} / \mathrm{dL})$ & $12.5 \pm 1.0(10.0-15.2)$ & $12.4 \pm 1.3(10.1-14.5)$ & 0.688 \\
\hline
\end{tabular}

Values are presented as mean \pm standard deviation (range).

SBTKA: simultaneous bilateral total knee arthroplasty, BMl: body mass index, ASA: American Society of Anesthesiologists.

${ }^{*} p$-value $<0.05$ indicates statistical significance. 
Rattanaprichavej et al. Closed- versus Open-Box Prosthesis

Clinics in Orthopedic Surgery • Vol. 11, No. 4, $2019 \bullet$ www.ecios.org

Table 2. Comparison of Perioperative Characteristics between the Closed-Box and Open-Box SBTKA

\begin{tabular}{|c|c|c|c|}
\hline Variable & Closed-box SBTKA & Open-box SBTKA & $p$-value \\
\hline Total operative time (min) & $123.1 \pm 20.4$ & $125.5 \pm 21.1$ & 0.874 \\
\hline \multicolumn{4}{|l|}{ Drain output (mL) } \\
\hline Day 1 & $331.1 \pm 181.0$ & $380.7 \pm 253.6$ & 0.246 \\
\hline Day 2 & $240.4 \pm 121.8$ & $263.1 \pm 188.5$ & 0.465 \\
\hline Calculated blood loss (mL) & $874.1 \pm 243.3$ & $1,015.2 \pm 356.8$ & $0.018^{*}$ \\
\hline Blood transfusion rate (\%) & 24.1 & 38.5 & 0.107 \\
\hline Length of stay (day) & $7.2 \pm 1.2$ & $7.3 \pm 1.4$ & 0.478 \\
\hline
\end{tabular}

Values are presented as mean \pm standard deviation.

SBTKA: simultaneous bilateral total knee arthroplasty.

${ }^{*} p$-value $<0.05$ indicates statistical significance.

and LOS were not different between closed-box and openbox TKAs (Table 2). The DOs on day 1 and 2 in open-box SBTKA were greater than those in closed-box SBTKA, but there was no statistical significance. The CBL in closedbox SBTKA was significantly lower than that in open-box SBTKA $(874.1 \pm 243.3$ vs. $1,015.2 \pm 356.8 \mathrm{~mL} ; p=0.02)$ as presented in Table 2.

In multiple linear regression analysis, controlling for age, BMI, ASA, preoperative $\mathrm{Hb}$, TOT, and type of knee prosthesis, the CBL of the closed-box was $135.2 \mathrm{~mL}$ less than that of the open-box TKA (95\% confidence interval [CI], -215.3 to $-55.2 ; p=0.001)$. For each additional minute of TOT, $3.8 \mathrm{~mL}$ (95\% CI, 1.8 to $5.8 ; p<0.01$ ) of blood loss was anticipated while age, BMI, and ASA had no significant effect on the CBL.

The blood transfusion rates of closed-box and openbox TKA groups were $24.1 \%$ and $38.5 \%$, respectively ( $p=$ 0.11 ). With multiple logistic regression analysis, adjusting for age, BMI, ASA, preoperative $\mathrm{Hb}$, TOT, and type of knee prosthesis, $71 \%$ reduction of blood transfusion probability was predicted for each additional $\mathrm{mg} / \mathrm{dL}$ of preoperative $\mathrm{Hb}$ $(p<0.001)$, while other factors had no significant effect.

\section{DISCUSSION}

Blood loss is an inevitable morbidity after TKA and it is more substantial in patients with SBTKA. ${ }^{17)}$ Although ongoing bleeding from venous sinus of trimmed cancellous bone is responsible for blood loss after UTKA, ${ }^{4,14,18)}$ studies that directly analyze the perioperative blood loss according to different prostheses are limited. Therefore, our study aims to differentiate the effect of different knee prostheses on blood loss in patients undergoing SBTKA.
In the current study, the closed-box SBTKA could significantly reduce CBL by $135.23 \mathrm{~mL}$ when compared to open-box SBTKA, even though patellar resurfacing was performed routinely in the closed-box group. MahringerKunz et al. ${ }^{7)}$ assessed a series of 473 patients who underwent either PS or CR UTKA. They reported the CBL was significantly lower (by $46 \mathrm{~mL}$ ) in CR UTKA. Cankaya et al. ${ }^{14)}$ also compared 50 CR UTKAs and 50 PS UTKAs and found that DO was $43 \mathrm{~mL}$ lower and CBL was $74 \mathrm{~mL}$ lower in CR UTKA. We agree with these previous studies: the area of the trimmed cancellous surface that was uncovered by prosthesis may be responsible for differences in CBL between different knee prosthetic designs, ${ }^{5-7,14)}$ because the total DO was $72.36 \mathrm{~mL}$ less in closed-box TKA. However, the blood transfusion rate was not significantly different according to the selection of knee prosthesis, even in our SBTKA setting. Although an occlusion of the femoral opening hole with the bone plug was applied to reduce the blood loss, Torres-Claramunt et al. ${ }^{19)}$ reported a similar outcome regarding $\mathrm{DO}$, postoperative $\mathrm{Hb}$ level, and transfusion rate when an open-box prosthesis was implanted with or without a femoral bone plug. In addition, Moo et $\mathrm{al}^{20)}$ recently investigated the sealant effect of bone wax to reduce blood loss from exposing a trimmed cancellous surface in UTKA. They demonstrated less bleeding from the occluded nail hole and a significant decrease of total blood loss in the bone wax group than in the control group ( 987.9 vs. $1,183.5 \mathrm{~mL}$ ). Other strategies including plateletrich plasma and platelet gel applied to the bleeding surface before TKA wound closure could also reduce blood loss after the index surgery. ${ }^{21,22)}$ Therefore, special techniques that control any bleeding surface may further minimize the perioperative blood loss regardless of femoral hole oc- 
Rattanaprichavej et al. Closed- versus Open-Box Prosthesis

Clinics in Orthopedic Surgery • Vol. 11, No. 4, $2019 \bullet$ www.ecios.org

clusion.

Some pre- and perioperative risk factors including age, sex, race, BMI, preoperative $\mathrm{Hb}$ level, comorbidity, and duration of operation have been reported to affect the CBL and postoperative blood transfusion of UTKA. ${ }^{8,12,13,23,24)}$ Preoperative $\mathrm{Hb}$ level is considered as a major predictor because lower preoperative $\mathrm{Hb}$ level may affect the gradient between baseline and physician transfusion trigger. ${ }^{25)}$ Patients aged more than 85 years have 3.5 times higher blood transfusion rate than do younger patients. Compromise of postoperative hematopoietic activity due to advanced age may be an explanation. ${ }^{12)}$ Prolonged operation time may be a result of difficult and extensive surgical dissection of the procedure and lead to more blood loss. ${ }^{13,25)}$ A higher ASA score reflecting patient's comorbidity may confound clotting cascade. ${ }^{8,12)}$ Furthermore, male and Caucasian patients have a lower risk for blood transfusion after UTKA as shown in several studies. ${ }^{8,12,13)}$ According to our study, each additional minute of TOT would result in $3.75 \mathrm{~mL}$ more CBL in SBTKA, but it had no effect on the blood transfusion requirement. The preoperative $\mathrm{Hb}$ level was the only factor affecting the blood transfusion rate with $71 \%$ less likely to get a blood transfusion for each additional $\mathrm{mg} / \mathrm{dL}$ of preoperative $\mathrm{Hb}$. Various blood-preserving techniques aimed at decreasing the complications from a blood transfusion have been described in contemporary orthopedic literature. Among these techniques, administration of TXA is one of the greatest avenues to decrease blood loss in SBTKA without increasing the risk of a thromboembolic event. ${ }^{26-29)}$ Chen et al. ${ }^{26)}$ revealed the effectiveness of IV TXA administration in SBTKA. Their results showed a significant decrease in blood loss (1,739.5 vs. $2,392.9 \mathrm{~mL}$ ), DO (245.3 vs. 613.4 $\mathrm{mL})$, transfusion rate (60\% vs. $96.7 \%)$, and the average number of transfused blood units (1.9 vs. 4.1 units) with IV TXA when compared to SBTKA without TXA. Bagsby et al., ${ }^{27)}$ Karaaslan et al., ${ }^{28)}$ and Aggarwal et al. ${ }^{29)}$ all showed results that support the advantage of topical and IV administration of TXA in SBTKA patients. The effect of TXA may be one of the reasons why age, BMI, and ASA had an insignificant effect on CBL and blood transfusion rate in our study.

There are some limitations to the present study. First, our study group consisted of predominantly women. Therefore, we could not determine the effect of gender on $\mathrm{CBL}$ and blood transfusion rate in the analysis. Neverthe- less, females have been associated with significantly less blood loss after UTKA when compared to males. ${ }^{23,24)} \mathrm{Sec}-$ ond, we prefer to routinely resurface the patella in closedbox SBTKA but not in open-box SBTKA. Despite this difference, closed-box SBTKA significantly reduced the CBL and DO when compared to open-box SBTKA. To the best of our knowledge, bleeding from resurfacing of the patella has not been well demonstrated and might not have confounded our result. Third, unlike other studies, our investigation found an insignificant impact of ASA, BMI, and age on CBL and postoperative blood transfusion. This may be due to the effect of TXA and narrow distribution of our study population. Fourth, our transfusion threshold according to the institutional protocol is higher than the conventional transfusion trigger. The difference in preoperative $\mathrm{Hb}$ and transfusion cut-off level may have caused different outcomes.

The use of closed- and open-box knee prostheses could result in a significant difference in blood loss in SBTKA. Prolonged operative time also significantly increased CBL. Therefore, strategies to control any bleeding surface and shorten operative duration may be considered if blood loss is of special concern. The preoperative $\mathrm{Hb}$ was the only factor that affects the probability of blood transfusion in SBTKA.

\section{CONFLICT OF INTEREST}

No potential conflict of interest relevant to this article was reported.

\section{ACKNOWLEDGEMENTS}

We thank Atthakorn Jarusriwanna, MD (Faculty of Medicine, Naresuan University, Phitsanulok, Thailand), Nattharut Chaibhuddanugul, MD (Faculty of Medicine, Naresuan University, Phitsanulok, Thailand), Bhuwad Chinwatanawongwan, MD (Faculty of Medicine, Naresuan University, Phitsanulok, Thailand), Pariphat Chompoonutprapa, MD (Faculty of Medicine, Naresuan University, Phitsanulok, Thailand), Edward B. Rasor, Richard M. Glover (Faculty of Humanity, Naresuan University, Phitsanulok, Thailand), and Waykin Nopanitaya, PhD (Faculty of Medicine, Naresuan University, Phitsanulok, Thailand) for their technical assistance. 
Rattanaprichavej et al. Closed- versus Open-Box Prosthesis

Clinics in Orthopedic Surgery • Vol. 11, No. 4, $2019 \bullet$ www.ecios.org

\section{REFERENCES}

1. Walmsley P, Murray A, Brenkel IJ. The practice of bilateral, simultaneous total knee replacement in Scotland over the last decade: data from the Scottish Arthroplasty Project. Knee. 2006;13(2):102-5.

2. Kulshrestha V, Kumar S, Datta B, Sinha VK, Mittal G. Ninety-day morbidity and mortality in risk-screened and optimized patients undergoing two-team fast-track simultaneous bilateral TKA compared with unilateral TKA-A prospective study. J Arthroplasty. 2018;33(3):752-60.

3. Fu D, Li G, Chen K, Zeng H, Zhang X, Cai Z. Comparison of clinical outcome between simultaneous-bilateral and staged-bilateral total knee arthroplasty: a systematic review of retrospective studies. J Arthroplasty. 2013;28(7):1141-7.

4. Mylod AG Jr, France MP, Muser DE, Parsons JR. Perioperative blood loss associated with total knee arthroplasty: a comparison of procedures performed with and without cementing. J Bone Joint Surg Am. 1990;72(7):1010-2.

5. Raut VV, Stone MH, Wroblewski BM. Reduction of postoperative blood loss after press-fit condylar knee arthroplasty with use of a femoral intramedullary plug. J Bone Joint Surg Am. 1993;75(9):1356-7.

6. Levy O, Martinowitz U, Oran A, Tauber C, Horoszowski H. The use of fibrin tissue adhesive to reduce blood loss and the need for blood transfusion after total knee arthroplasty: a prospective, randomized, multicenter study. J Bone Joint Surg Am. 1999;81(11):1580-8.

7. Mahringer-Kunz A, Efe T, Fuchs-Winkelmann S, Schuttler KF, Paletta JR, Heyse TJ. Bleeding in TKA: posterior stabilized vs. cruciate retaining. Arch Orthop Trauma Surg. 2015;135(6):867-70.

8. Slover J, Lavery JA, Schwarzkopf R, Iorio R, Bosco J, Gold HT. Incidence and risk factors for blood transfusion in total joint arthroplasty: analysis of a statewide database. J Arthroplasty. 2017;32(9):2684-7.e1.

9. Jenny JY, Trojani C, Prudhon JL, et al. Simultaneous bilateral total knee arthroplasty: a multicenter feasibility study. Orthop Traumatol Surg Res. 2013;99(2):191-5.

10. Friedman R, Homering M, Holberg G, Berkowitz SD. Allogeneic blood transfusions and postoperative infections after total hip or knee arthroplasty. J Bone Joint Surg Am. 2014;96(4):272-8.

11. Silliman CC, Fung YL, Ball JB, Khan SY. Transfusion-related acute lung injury (TRALI): current concepts and misconceptions. Blood Rev. 2009;23(6):245-55.

12. Yoshihara H, Yoneoka D. Predictors of allogeneic blood transfusion in total hip and knee arthroplasty in the United States, 2000-2009. J Arthroplasty. 2014;29(9):1736-40.
13. Frisch NB, Wessell NM, Charters MA, Yu S, Jeffries JJ, Silverton CD. Predictors and complications of blood transfusion in total hip and knee arthroplasty. J Arthroplasty. 2014;29(9 Suppl):189-92.

14. Cankaya D, Ozkurt B, Aydin C, Tabak AY. No difference in blood loss between posterior-cruciate-ligamentretaining and posterior-cruciate-ligament-stabilized total knee arthroplasties. Knee Surg Sports Traumatol Arthrosc. 2014;22(8):1865-9.

15. Nadler SB, Hidalgo JH, Bloch T. Prediction of blood volume in normal human adults. Surgery. 1962;51(2):224-32.

16. Chen JY, Chin PL, Moo IH, et al. Intravenous versus intraarticular tranexamic acid in total knee arthroplasty: a double-blinded randomised controlled noninferiority trial. Knee. 2016;23(1):152-6.

17. Qi Y, Tie K, Wang H, et al. Perioperative comparison of blood loss and complications between simultaneous bilateral and unilateral total knee arthroplasty for knee osteoarthritis. Knee. 2017;24(6):1422-7.

18. Kalairajah Y, Simpson D, Cossey AJ, Verrall GM, Spriggins AJ. Blood loss after total knee replacement: effects of computer-assisted surgery. J Bone Joint Surg Br. 2005;87(11):1480-2.

19. Torres-Claramunt R, Hinarejos P, Perez-Prieto D, et al. Sealing of the intramedullar femoral canal in a TKA does not reduce postoperative blood loss: a randomized prospective study. Knee. 2014;21(4):853-7.

20. Moo IH, Chen JY, Pagkaliwaga EH, Tan SW, Poon KB. Bone wax is effective in reducing blood loss after total knee arthroplasty. J Arthroplasty. 2017;32(5):1483-7.

21. Aggarwal AK, Shashikanth VS, Marwaha N. Plateletrich plasma prevents blood loss and pain and enhances early functional outcome after total knee arthroplasty: a prospective randomised controlled study. Int Orthop. 2014;38(2):387-95.

22. Gardner MJ, Demetrakopoulos D, Klepchick PR, Mooar PA. The efficacy of autologous platelet gel in pain control and blood loss in total knee arthroplasty: an analysis of the haemoglobin, narcotic requirement and range of motion. Int Orthop. 2007;31(3):309-13.

23. Cushner FD, Friedman RJ. Blood loss in total knee arthroplasty. Clin Orthop Relat Res. 1991;(269):98-101.

24. Prasad N, Padmanabhan V, Mullaji A. Blood loss in total knee arthroplasty: an analysis of risk factors. Int Orthop. 2007;31(1):39-44.

25. Noticewala MS, Nyce JD, Wang W, Geller JA, Macaulay W. 
Rattanaprichavej et al. Closed- versus Open-Box Prosthesis

Clinics in Orthopedic Surgery • Vol. 11, No. 4, $2019 \bullet$ www.ecios.org

Predicting need for allogeneic transfusion after total knee arthroplasty. J Arthroplasty. 2012;27(6):961-7.

26. Chen X, Cao X, Yang C, Guo K, Zhu Q, Zhu J. Effectiveness and safety of fixed-dose tranexamic acid in simultaneous bilateral total knee arthroplasty: a randomized double-blind controlled trial. J Arthroplasty. 2016;31(11):2471-5.

27. Bagsby DT, Samujh CA, Vissing JL, Empson JA, Pomeroy DL, Malkani AL. Tranexamic acid decreases incidence of blood transfusion in simultaneous bilateral total knee arthroplasty. J Arthroplasty. 2015;30(12):2106-9.
28. Karaaslan F, Karaoglu S, Mermerkaya MU, Baktir A. Reducing blood loss in simultaneous bilateral total knee arthroplasty: combined intravenous-intra-articular tranexamic acid administration. A prospective randomized controlled trial. Knee. 2015;22(2):131-5.

29. Aggarwal AK, Singh N, Sudesh P. Topical vs intravenous tranexamic acid in reducing blood loss after bilateral total knee arthroplasty: a prospective study. J Arthroplasty. 2016;31(7):1442-8. 